\title{
Optimal maintenance, repair, and replacement for Great Lakes marine diesels
}

\author{
A.N. Perakis \\ Department of Naval Architecture and Marine Engineering, The University of Michigan, \\ Ann Arbor, MI 48109, USA
}

B. İnözü

School of Naval Architecture and Marine Engineering, University of New Orleans, New Orleans, LA 70148, USA

Received March 1990; revised December 1990

\begin{abstract}
Reliability-based models are developed and implemented to rationalize current winter layup replacement practices for one-engine and two-engine Great Lakes marine diesel ships. A semi-Markov competing-process approach is used in our models, where the age-dependent system failure behavior is treated as a race among engine components. Howard's one-set competing process model is implemented and extended to two sets of competing processes. An efficient enumeration procedure is presented to select the replacement policy which produces the minimum expected cost for the operating season. Computer codes are developed using the above models, and several examples are considered. Sensitivity analyses are performed for several parameters for which we have insufficient or no information from the industry to see their influence on the minimal expected costs and corresponding replacement policies.
\end{abstract}

Keywords: Maintenance, reliability, optimization, stochastic processes

\section{Introduction and outline}

The aircraft, electronics, and automotive industries, as well as the US Air Force, Army and Navy have been increasingly applying the results of reliability analysis to optimize maintenance and replacement decisions for more than 30 years. However, the marine transportation industry has been reluctant to adopt these techniques, as apparent from our informal survey of the maintenance, repair and replacement practices of most US Great Lakes operators whose vessels are equipped with diesel engines [5]. The survey showed that among Great Lakes shipping companies utilizing marine diesel engines, a few do not keep maintenance/repair records at all, and are usually content to follow manufacturer's recommendations. There are some companies which keep manual records of failures, repairs and replacements. In both cases, these records are used in a subsequent decisions in a rather ad hoc way. A small number of companies have tried using a computerized database management or decision support system [4].

In general, Great Lakes diesel operators tend to over-maintain and over-replace. The main reasons for this over-maintenance seem to be the conservative nature of the manufacturer's recommendations and the desire to avoid disabling failures during the short operating season. On the part of the manufacturer, this conservative approach could aim at the avoidance of legal problems related to the performance of their engines. Besides, Coast Guard and classification societies often require excessive numbers of spares to be carried on board the ships, thus further increasing the respective costs. 
We focus on two types of systems (engine room configurations), one with one engine only and another with two identical engines. Our prototype engine is a Colt-Pielstick PC2-400 series marine diesel engine and the auxiliaries that are critical to its operation. These engines are four-stroke, V-type, 16-cylinder, rated at $500 \mathrm{hp} /$ cylinder, at $514 \mathrm{rpm}$.

Access to extensive failure data was the main reason for selecting this particular engine. We have been receiving continuously updated failure data from Interlake Steamship $\mathrm{Co}$. which uses a database management system that was developed at the Department of Naval Architecture and Marine Engineering of The University of Michigan [3], using the 'Knowledgeman' database management software. This system has been in use by that company since January 1985 . However, the records developed also contain information obtained manually before that time. The data at our disposal contain information pertaining to engine failures since 1976 for six engines in three ships.

Our system's operating conditions are as follows:

- The Great Lakes are closed to navigation during the winter months. Winter layup approximately starts on January 5 and ends on March 20.

- The existence of the winter lay-up in Great Lakes shipping allows (and also encourages) operators to do most of their maintenance and replacement work during the non-operating season.

- Great Lakes operators prefer replacing several working engine components during the winter period, since it is very important to minimize down time during the operating season.

- Great Lakes ships frequently operate in restricted waters, close to major equipment supply centers, and may reach several such ports or centers every day. Therefore, it is much easier for them to order and receive spare parts than it is, for example, for a tanker routinely traveling between the Persian Gulf and Japan [5].

We have discussed basic characteristics of existing repair, replacement and maintenance models and presented the results of our literature survey on these models in [2].

The remainder of this paper is organized as follows:

In Section 2, we state and discuss our assumptions. In Section 3, we present our models for one- and two-engine systems. In Section 4, we present an optimization algorithm for selecting the best replacement policy using these models. In Section 5, we discuss the implementation of our models in our computer codes for two-engine systems. In Section 6, we focus on our two-engine model, and we present and discuss the sensitivity of the results for variations in the values of system parameters. In Section 7, we summarize our conclusions.

\section{General assumptions}

We make the following assumptions, valid for both one- and two-engine systems:

(1) We consider a finite time horizon problem, equal in length to the annual operating season.

(2) The operating season starts at time zero, and ends at time $T_{0}$ in terms of engine hours. It is not required that all system components are 'as good as new' at time zero. However, all system components should be operational at time zero. At the beginning of each winter lay-up, the decision maker reviews the condition of all components in question and chooses one of two possible options for each component.

(a) replace;

(b) do nothing.

Reconditioning could be added as a third option. Due to insufficient information on current reconditioning practices, we have not included this option. Necessary and sufficient conditions for reconditioning depend on the wearout of the component in question. In general, a mechanical component's wearout is proportional to its age. These age-wearout relations for our system components can be examined. When these relations are developed, we can include reconditioning as another option.

(3) We want to evaluate whether to replace or not each system component at the beginning of each winter lay-up. 
(4) During the operating season, the system operates intermittently. The system should succesfully complete $n$ missions during the annual operating season. Each mission refers to a one-way trip from one port to another port.

(5) At the end of each mission (voyage) period, there is an 'off-period' (port period), during which the system does not operate. Mission durations and port times are assumed to be constant and known.

(6) Component failure times are statistically independent.

(7) At the beginning of an operating season, the failure PDF's for all components are known.

(8) Two or more components of a one- or two-engine system cannot fail at the same time instant.

(9) Repair and replacement costs, $C_{i r}$, are assumed to be of the form

$$
C_{i \mathrm{r}}=Y_{i} l_{i}+b_{i}
$$

where $Y_{i}$ is the repair/replacement cost per unit time, $b_{i}$ is the fixed repair/replacement cost, $l_{i}$ is the required time to complete repair/replacement of component $i$. $Y_{i}$ corresponds to opportunity costs and repair/replacement crew costs, whereas $b_{i}$ corresponds to fixed repair/replacement costs, e.g. transportation cost of bringing a repair/replacement crew to the ship, along with the cost of repair/replacement materials for the $i$-th component.

(10) We consider deterministic repair/replacement times, as suggested by Interlake Steamship company engineers based on their experience.

(11) Repairs which are carried out during the operating season will bring the component to the 'as good as just before failure' condition (minimal repair assumption).

(12) When the system is not operational, the system neither fails nor deteriorates (No aging takes place during winter lay-up and port stays).

(13) The ages of the system components are measured by the total use-time of the components, e.g. in engine hours.

(14) Preventive replacements will not be permitted while the system is in use.

(15) During a trip between any two ports, the system experiences at most two failures.

Other assumptions, valid only for one- or only for two-engine systems, are presented in the following sections.

\section{Models}

We consider two systems: one for a ship equipped with one engine only and another for a two-engine ship. The failure characteristics of these systems are summarized below.

If ' $\mathrm{A}$ ' denotes the age (in terms of engine hours) of a component, and ' $u$ ' denotes the time to failure of an item that survived $A$ hours then the component's residual reliability, $R_{\text {res }}$, is

$$
R_{\text {res }}(u)=\frac{R(A+u)}{R(A)}
$$

where $R(\cdot)$ is the reliability of the component.

Since the ages of our engine components play a very important role in state transitions, the changes of system states are assumed to follow a semi-Markov process. We will first focus on a one-engine system. For such a system, a semi-Markov process is defined as follows:

Consider a stochastic process with states $0,1, \ldots$, such that, whenever it is a state $i$ :

(i) The next state it will enter is state $j$ with probability $P_{i j}$, referred to as the transition probability.

(ii) Given that the next state the system will enter is state $j$, the residence time in state $i$ has probability distribution $F_{i j}(t)$.

If we let $Z(t)$ denote the state at time $t$, then stochastic process $\{Z(t), t \geqslant 0\}$ is called a semi-Markov process. For predictions of the future, it is necessary to know not only the present state (which is the case for continuous time Markov processes) but also the residence time in that state, which is a random variable 
whose PDF depends on the transition. Hence a semi-Markov process does not possess the property that "the future is independent of the past when the present state is given" [6].

Our engine has $N$ components. For a one engine system, if 0 denotes the operating state, and ' $j$ ' denotes the failed engine states $(j=1,2, \ldots, N)$, then $P_{j 0}=1$. The failure of each component causes an engine failure. In other words, we can characterize this failure process as a race among engine components. In this 'race', components are literally competing with each other in terms of failing. The component which fails first causes engine failure. This system state transition model is called the Competing Process Model [1].

We assumed that the failure times of engine components are independent of each other. Each component has a different failure time PDF. We define the transition probability $P_{j}\left(P_{j} \equiv P_{i j}\right.$ for $\left.i=0\right)$ to be the probability that the engine failure is due to the failure of the $j$-th component $(j=1,2, \ldots, N)$. Alternatively, we can define the $P_{j}$ 's as follows:

$$
\begin{aligned}
& P_{1}=\operatorname{Prob}\left[\bigcap_{j \neq 1}^{N}\left\{\Xi_{(1)}<\xi_{(j)}\right\}\right], \\
& P_{2}=\operatorname{Prob}\left[\bigcap_{j \neq 2}^{N}\left\{\Xi_{(2)}<\xi_{(j)}\right\}\right], \\
& P_{N}=\operatorname{Prob}\left[\bigcap_{j \neq N}^{N}\left\{\Xi_{(N)}<\xi_{(j)}\right\}\right],
\end{aligned}
$$

where $\Xi_{(j)}$ denotes the random variable defined as component $j$ 's failure time and $\xi_{(j)}$ denotes an actual value of this random variable.

The transition probabilities, which depend on ages of all components, can be calculated from

$$
P_{j}=\int_{0}^{\infty} \mathrm{d} \xi g_{j}(\xi) \prod_{k \neq j} R_{k}(\xi)
$$

where $g_{j}$ denotes the residual life time PDF of the $j$-th component, and $R_{k}(\xi)$ denotes the residual reliability of component $k$ at time $\xi$. $P_{j}$ depends on the ages of all system components [1]. The residence time PDF, $f_{j}$, which is the system's failure time PDF (due to component $j$ ), is

$$
f_{j}(\xi)=P_{j}^{-1}\left[g_{j}(\xi) \prod_{k=1, k \neq j}^{N} R_{k}(\xi)\right] .
$$

We will first develop an expected cost model for one-engine vessels (one-engine model). We will then use the results of that model to construct a two-engine system vessel (two-engine model). Our two-engine system consists of two identical engines. However, each engine may have experienced different (if any) failures during past operation, and as a result, corresponding components of each engine may have been treated differently. For example, piston \#3 of Engine I may have been replaced due to a failure, whereas piston \# 3 of Engine II may have been working satisfactorily and may have never been replaced. Since our system is composed of mechanical components which wear out with operation, the hazard rates of these components are not constant, as apparent from our data. Hence the reliability functions of engine components are age dependent. Therefore, Engine I and Engine II are not necessarily identical from a reliability standpoint (not even, for example, piston \#3 and piston \#5 of the same engine).

\subsection{One-engine model}

We assume that a one-engine system has $N+1$ states, one 'up' state, denoted by ' 0 ' and $N$ 'down' states. If component $i$ of the engine fails, this engine will make a transition to down state $i(i=1,2, \ldots, N)$. 
Our ship will stop immediately after an engine failure and cannot be operated until the (failed) engine is brought back to the operating condition.

At time zero, the engine starts operating. At time $T_{0}$, which marks the end of the operating season, we may observe one of the following two cases:

Case I. The engine has stayed in state 0 throughout the interval $\left(0, T_{0}\right)$; e.g., the engine may have not experienced any failures during the entire operating season.

Case II. The engine has experienced at least one state transition during $\left(0, T_{0}\right)$; e.g., the system has failed at time $\xi, \xi \in\left(0, T_{0}\right)$, where $\xi$ is a random variable [1].

Define $\vartheta_{0}(t)$ as the expected value of the total cost incurred during an operating season of length $t$ (in terms of engine hours), if the system is in working order at the beginning of this season.

The expected value of total cost incurred by the system is

$$
\begin{aligned}
& \vartheta_{0}(t)=\sum_{j=1}^{N} P_{j} R_{j}(t)\left[y_{0} t+\vartheta_{0}(0)\right] \\
& +\sum_{j=1}^{N} P_{j} \int_{0}^{t} \mathrm{~d} \xi f_{j}(\xi)\left[y_{0} \xi+b_{j}+y_{j} l_{j}+\vartheta_{0}\left(t-\xi-l_{j}\right)\right] \text { for } t>l_{j} .
\end{aligned}
$$

\section{Boundary conditions.}

For $t<0, \vartheta_{0}(t)=0$.

For $0 \leq t<l_{j}$,

$$
\begin{aligned}
\vartheta_{0}(t)= & \sum_{j=1}^{N} P_{j} R_{j}(t)\left[y_{0} t+\vartheta_{0}(0)\right] \\
& +\sum_{j=1}^{N} P_{j} \int_{0}^{t} \mathrm{~d} \xi f_{j}(\xi)\left[y_{0} \xi+b_{j}+y_{j}(t-\xi)+\vartheta_{0}(0)+\left(l_{j}-t+\xi\right) y_{j}^{*}\right],
\end{aligned}
$$

where $y_{j}^{*}$ is only the repair cost per unit residence time incurred during an engine's stay at failure state $j$, whereas $y_{j}$ is the repair and opportunity costs.

The first summations in (3) and (4) are the expected costs incurred for case I: Since nothing fails during the operating season, the only cost is the operating costs, $y_{0} t$. At the end of the season, we may also incur a terminal cost, $\vartheta_{0}(0)$. The second summations are the expected costs incurred for case II: The engine operates for $\xi$ time units, during which the system incurs only operating costs. At time $\xi$, component $j$ fails, incurring a fixed cost, $b_{j}$, and a variable cost, $y_{j}$, which includes both opportunity costs and repair (or replacement) costs.

Equations (3) and (4) can be solved recursively, starting with $t=0$ and increasing $t$ by $\Delta t$ until we reach $t=T_{0}$. Details of this solution process are given in Section 4 .

\subsection{Two-engine model}

With two engines, if one of the engines fails at time $\xi_{1}$, we assume that the ship will continue sailing with only one engine working, producing less power than both engines. If only one engine fails during a mission, until the ship comes to the next port, corrective measures will not be taken to return the failed engine to the operating state. However, there is a non-zero probability that the second engine will also fail, at time $\xi_{2}, \xi_{1}<\xi_{2}<t_{p 1}$, before the first engine is fixed, where $t_{p 1}$ is the delayed arrival time at the next port if only one engine fails. In this case, we need to examine the repair/replacement times (TTR's) of both Engines I and II. We given repair/replacement priority to the engine whose TTR is smaller. As a result, we may observe one of the five cases $(\mathrm{A}, \mathrm{B}, \mathrm{C}, \mathrm{D}, \mathrm{E})$ shown in Figures $1-3$ at time $T_{0}$; i.e., at the end of operating season. Before we explain these cases, we next define some variables and system states as follows. 


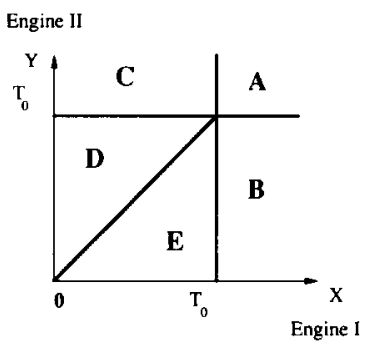

Figure 1. Locations of regions for a two-engine system

Region A: $\mathrm{X}>\mathrm{T}_{0}, \mathrm{Y}>\mathrm{T}_{0}$

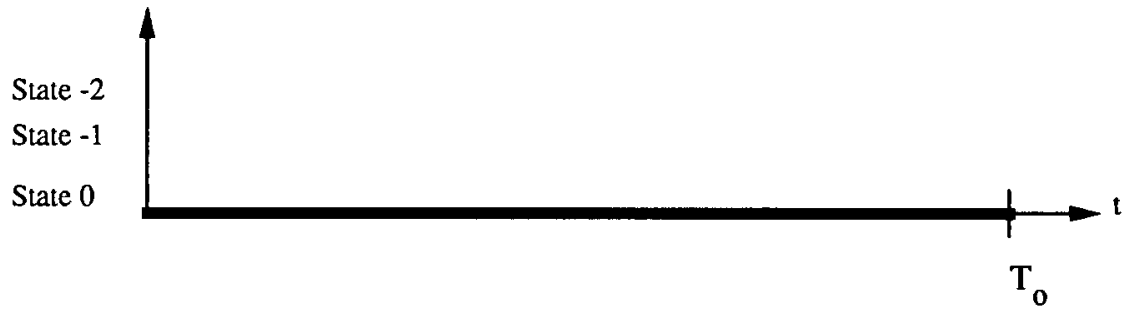

Region $\mathrm{B}: \mathrm{X}>\mathrm{T}_{0}, \mathrm{~T}_{0}>\mathrm{Y}>0$

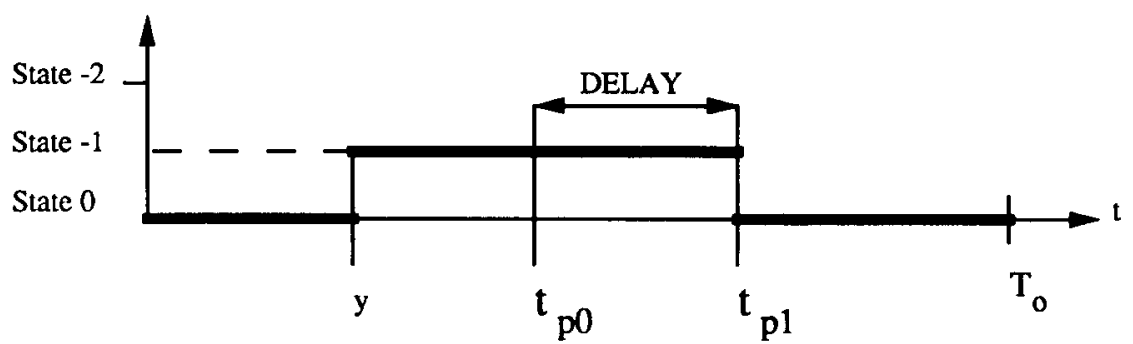

Region $\mathrm{C}: \mathrm{Y}>\mathrm{T}_{0}, \mathrm{~T}_{0}>\mathrm{X}>0$

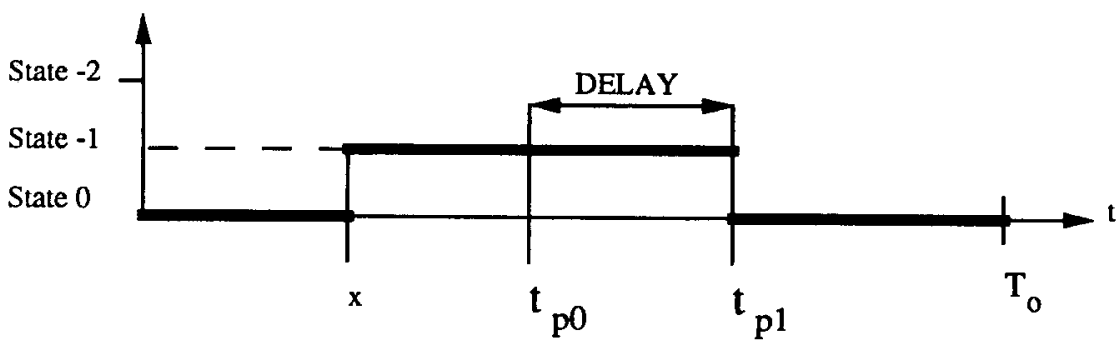

Figure 2. State trajectory of a two-engine system for regions A, B, C 
Region D: $\mathrm{Y}>\mathrm{X}, \quad \mathrm{X}<\mathrm{T}_{0}, \quad \mathrm{Y}<\mathrm{T}_{0}$

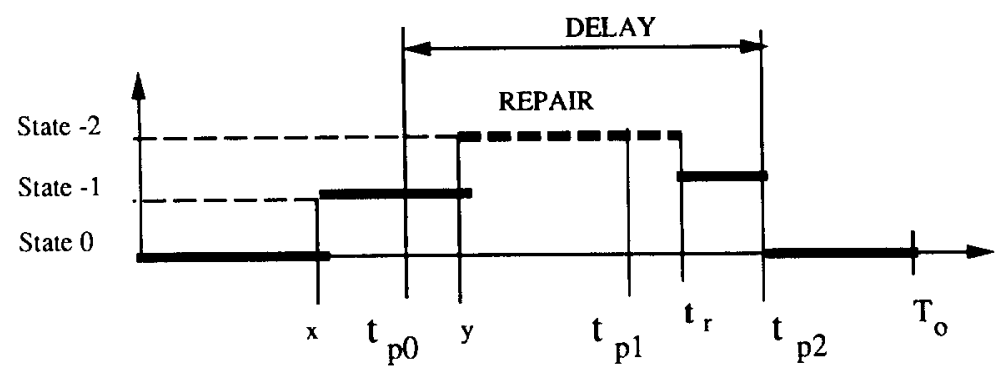

Region $\mathrm{E}: \mathrm{X}>\mathrm{Y}, \mathrm{X}<\mathrm{T}_{0}, \mathrm{Y}<\mathrm{T}_{0}$

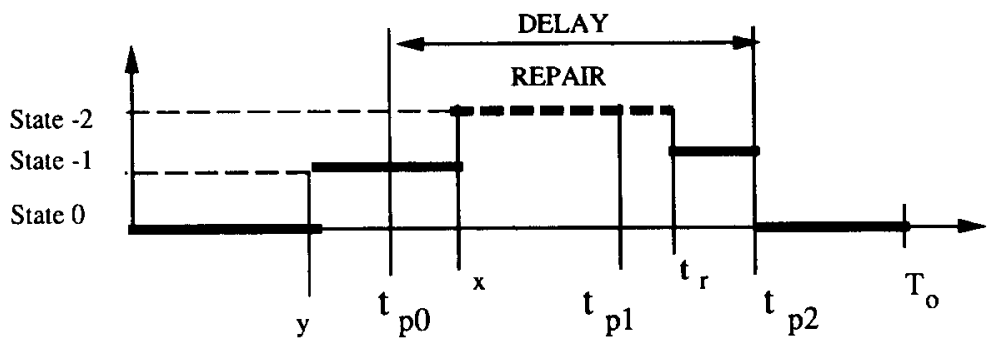

Figure 3. State trajectory of a two-engine system for regions D, E

Let random variable $X(X>0)$ denote the first failure time of Engine I and random variable $Y(Y>0)$ denote the first failure time of Engine II.

We have three possible general system states.

State ' $O$ '. Both engines are operating properly.

State ' $-l$ '. One engine has failed and the other is operating (properly).

State ' -2 '. Both engines have failed.

Let subscripts $i$ and $j$ indicate the source of engine failure for Engines I and II respectively; i.e., they show which component caused the engine failure. Transition probabilites are defined exactly as in the one-engine case. For the two-engine case, given that a failure occurs in each engine,

$$
\begin{aligned}
& \sum_{i=1}^{N_{\mathrm{I}}} P_{\mathrm{I} i}=1, \quad P_{\mathrm{I} i} \geq 0, \\
& \sum_{j=1}^{N_{\mathrm{II}}} P_{\mathrm{II} j}=1, \quad P_{\mathrm{II} j} \geq 0,
\end{aligned}
$$

where $P_{\mathrm{I} i}$ and $P_{\mathrm{II} j}$ refer to the transition probabilities of Engines I and II, respectively. $N_{\mathrm{I}}$ and $N_{\mathrm{II}}$ are the number of components of Engines I and II. The five regions shown in Figure 1 correspond to the following five cases:

Case $A$. They system stays in State 0 throughout the interval $\left(0, T_{0}\right)$; i.e., the system does not experience any failures during the operating season.

Case $B$. Engine II fails at $Y$, and the ship continues sailing to the next port with only Engine I operating. The ship waits at the port until Engine II is fixed.

Case $C$. Engine I fails at $X$, and the ship continues sailing to the next port with only Engine II operating. The ship waits at the port until Engine I is fixed. 
Case D. Engine II fails at $Y$, the ship continues sailing with only Engine I operating. However, Engine I also fails at $Y$ before the ship arrives at the next port. As a result either Engine I or Engine II is fixed at sea, depending on repair/replacement times and associated costs. When one of the engines is fixed, the ship continues sailing with only the repaired engine operating and arrives at the next port. The ship waits at the port until the other failed engine is fixed $\left(0<X<T_{0}, 0<Y<T_{0}, X>Y\right)$.

Case $E$. Engine I fails at $X$, the ship continues sailing with only Engine II operating. However, Engine II also fails at $Y$ before the ship arrives at the next port. As a result, either Engine I or Engine II is fixed at sea, again depending on repair/replacement times and associated costs. When one of the engines is repaired, the ship continues sailing with only the repaired engine operating and arrives at the next port. Similar to Case $\mathrm{D}$, the ship waits at the port until the other engine is fixed $\left(0<X<T_{0}, 0<Y<T_{0}, Y>X\right)$.

The expected cost incurred during an operating season whose duration is $t$, is

$$
V(t)=\mathrm{E}[C(x, y)]=\int_{0}^{\infty} \int_{0}^{\infty} C(x, y) f_{X Y}(x, y) \mathrm{d} x \mathrm{~d} y,
$$

where $C(x, y)$ represents the cost incurred by the system during a time interval of length $t$, and $f_{X Y}(x, y)$ represents the joint PDF of $X$ and $Y$, the failure times of Engines I and II, respectively.

Since $X$ and $Y$ are assumed to be independent, we can write

$$
f_{X Y}(x, y)=f_{X}(x) f_{Y}(y)
$$

where the lifetime PDF's Engine I and II, respectively, are

$$
f_{X}(x)=\sum_{i=1}^{N_{\mathrm{I}}} P_{\mathrm{I} i} g_{i}(x), \quad f_{Y}(y)=\sum_{j=1}^{N_{\mathrm{ll}}} P_{\mathrm{Il} j} g_{j}(y) \text {. }
$$

We can rewrite (5) in terms of the regions shown in Figure 1 as follows:

$$
\begin{aligned}
\mathrm{E}[C(x, y)]= & \iint_{\mathrm{A}} C_{\mathrm{A}} f_{X}(x) f_{Y}(y) \mathrm{d} x \mathrm{~d} y+\iint_{\mathrm{B}} C_{\mathrm{B}}(x, y) f_{X}(x) f_{Y}(y) \mathrm{d} x \mathrm{~d} y \\
& +\iint_{\mathrm{C}} C_{\mathrm{C}}(x, y) f_{X}(x) f_{Y}(y) \mathrm{d} x \mathrm{~d} y+\iint_{\mathrm{D}} C_{\mathrm{D}}(x, y) f_{X}(x) f_{Y}(y) \mathrm{d} x \mathrm{~d} y \\
& +\iint_{\mathrm{E}} C_{\mathrm{E}}(x, y) f_{X}(x) f_{Y}(y) \mathrm{d} x \mathrm{~d} y .
\end{aligned}
$$

We can now calculate the cost incurred in each region separately. Let us denote by $t_{p 0}$ the scheduled arrival time at the next port, given that no failures occurred since the beginning of the operating season. Define $t_{p 1}$ as the delayed arrival time (in hrs.) at the next port, given that only one of the engines fails during the current voyage, and $t_{p 2}$ as the delayed arrival time (in hrs.) at the next port, given that both Engine I and Engine II fail during the current voyage. In the definitions of $t_{p 1}$ and $t_{p 2}$, it is also assumed that first system failures occurred during the current voyage.

The time horizon is divided into voyage intervals, whose lengths are all equal to $L_{o p}$. The following equations are valid for $t>0$.

Region $A$. In this case, nothing fails during the operating season. As a result, the system only incurs operating costs and a terminal cost. Hence $C_{\mathrm{A}}$ becomes

$$
C_{\mathrm{A}}=Y_{0} t+V(0) \text {. }
$$

Region $B$. In this case, both engines operate for $y$ units, incurring only operating costs, $Y_{0} y$. Then component $j$ of Engine II fails at time $y$, incurring a fixed cost, $b_{j}$, and a variable repair (or replacement) $\operatorname{cost} Y_{j} l_{j}$. The ship continues sailing with one engine, causing a delay of $t_{p 1}-t_{p 0}$ time units, incurring a fraction, $\eta$, of regular operating costs. The scheduled port stay may not be sufficient to complete the repair (or replacement) of component $j$, and a delay of an additional $l_{j}^{\prime}$ time units may be required. Both these 
delays result in an opportunity cost stated as $Y_{\mathrm{oc}}\left(t_{p 1}-t_{p 0}+l_{j}^{\prime}\right)$, where $Y_{\mathrm{oc}}$ is the opportunity cost per unit time. At time $t-t_{p 1}-l_{j}^{\prime}$, the ship will be ready to start a new trip with both engines operating. Hence the cost incurred in region $B$ is

$$
\begin{aligned}
C_{\mathrm{B}}(x, y) & =C_{\mathrm{B}}(y) \\
& =Y_{0} y+\sum_{j=1}^{N_{\mathrm{II}}} P_{\mathrm{II} j}\left[b_{j}+Y_{j} l_{j}\right]+\sum_{j=1}^{N_{\mathrm{II}}} P_{\mathrm{II} j}\left[Y_{\mathrm{oc}}\left(t_{p 1}-t_{p 0}+l_{j}^{\prime}\right)+\eta Y_{0}\left(t_{p 1}-y\right)+V\left(\tau_{\mathrm{B}}\right)\right],
\end{aligned}
$$

where $\tau_{\mathrm{B}}=t-t_{p 1}-l_{j}^{\prime}$ for $\mathrm{t}>\mathrm{t}_{\mathrm{p} 1}+l_{\mathrm{j}}^{\prime}$ and $\tau_{\mathrm{B}}=0$ for $t \leqslant t_{p 1}+l_{j}^{\prime}$.

Rearranging the terms gives

$$
C_{\mathrm{B}}(y)=\sum_{j=1}^{N_{\mathrm{II}}} P_{\mathrm{II} j}\left[b_{j}+Y_{j} l_{j}+Y_{\mathrm{oc}} l_{j}^{\prime}+V\left(\tau_{\mathrm{B}}\right)\right]+\left(Y_{\mathrm{oc}}+\eta Y_{0}\right) t_{p 1}+(1-\eta) Y_{0} y-Y_{\mathrm{oc}} t_{p 0},
$$

where

$$
t_{p 1}=y+\gamma\left(t_{p 0}-y\right)
$$

Region $C$. In this case, Engine I fails first at time $x$ and the ship keeps sailing with Engine II operating. The only difference between Cases B and C is that in Case C, Engine I fails instead of Engine II. The expected cost calculation here is basically the same as that of region B. By interchanging first failure time of Engine I, $x$, with $y$ and interchanging the related probabilities in (9), the cost incurred in region $\mathrm{C}$ can be calculated.

Region $D$. In this case, the system will incur regular operating costs until the failure of Engine I at time $x$. Then a fixed cost, $b_{i}$, and a variable cost, $Y_{i} l_{i}$, will be incurred. The ship will continue sailing with Engine II, until it also fails at time $y, 0<x<y<t$. During this time interval of length $y-x$, the system will incur a fraction, $\eta$, of operating costs. At time $y$, the system will incur a fixed cost, $b_{j}$, and the ship stops sailing. Then, depending on the associated costs, either Engine I or Engine II will be repaired (or the failed component replaced) at sea and this repair (or replacement) will be completed at time $t_{r}$. Finally, our ship will arrive at the port at time

$$
t_{p 2}=\gamma t_{p 0}+(1-\gamma) x+l_{k},
$$

where $l_{k}$ denotes the repair (or replacement) time of the component chosen for repair (or replacement) at sea.

We define the delay ratio, $\gamma$, as the ratio of the ship speed when both engines are operating over the ship speed when one engine is operating $(\gamma>1)$.

The repair (or replacement) time of the failed component which is left for port repair/replacement is denoted by $l_{m}$. The scheduled port stay may not be sufficient to complete the repair/replacement of component $m$. An additional $l_{m}^{\prime}$ time units may be required, causing a delay of $l_{m}^{\prime}$ time units. Total delay for this trip will be $t_{p 2}-t_{p 0}+l_{m}^{\prime}$, resulting in an opportunity cost $\mathrm{Y}_{\mathrm{oc}}\left(t_{p 2}-t_{p 0}+l_{m}^{\prime}\right)$.

At time $t-t_{p 2}-l_{m}^{\prime}$, the ship will again be ready to start a new trip with both engines operating. The expected cost at this point is denoted as $V\left(t-t_{p 2}-l_{m}^{\prime}\right)$. Hence the resulting total cost is as follows:

$$
C_{\mathrm{D}}(x, y)=Y_{0} x+\eta Y_{0}(y-x)+Y_{\mathrm{oc}}\left(y-t_{p 0}\right)+\sum_{i=1}^{N_{1}} P_{\mathrm{I} i}\left[b_{i}+Y_{i} l_{i}+E_{\mathrm{D}}(x, y)\right],
$$

where

$$
E_{\mathrm{D}}(x, y)=\sum_{j=1}^{N_{\mathrm{II}}} P_{\mathrm{II} j}\left[b_{j}+Y_{j} l_{j}+Y_{\mathrm{oc}}\left(t_{p 2}-y\right)+\eta Y_{0}\left(t_{p 2}-y-l_{k}\right)+V\left(\tau_{\mathrm{D}}\right)\right]
$$

and $\tau_{\mathrm{D}}=t-t_{p 2}-l_{m}^{\prime}$ for $t>t_{p 2}+l_{m}^{\prime}$ and $\tau_{\mathrm{D}}=0$ for $t<t_{p 2}+l_{m}^{\prime}$. 
$\mathrm{C}_{\mathrm{D}}(x, y)$ can be rearranged as follows:

$$
\begin{aligned}
C_{\mathrm{D}}(x, y)= & \sum_{i=1}^{N_{\mathrm{t}}} P_{\mathrm{l} i}\left[b_{i}+Y_{i} l_{i}\right]+\sum_{j=1}^{N_{\mathrm{II}}} P_{\mathrm{II} j}\left[b_{j}+Y_{j} l_{j}\right]+\sum_{i=1}^{N_{\mathrm{I}}} P_{\mathrm{I} i}\left(\sum_{j=1}^{N_{\mathrm{II}}} P_{\mathrm{IIj}}\left[Y_{\mathrm{oc}} t_{p 2}+V\left(\tau_{\mathrm{D}}\right)\right]\right) \\
& +\eta Y_{0}\left[\gamma t_{p 0}+(1-\gamma) x\right]+Y_{0} x(1-\eta)-Y_{\mathrm{oc}} t_{p 0} .
\end{aligned}
$$

This equation is a function of which component fails at $y$, but not a function of $y$ itself. Hence $C_{\mathrm{D}}(x, y)=C_{\mathrm{D}}(x)$.

Region $E$. In this case, Engine II fails first at time $y$ and the ship keeps sailing with Engine I operating, until Engine I fails at time $x, 0<y<x<t$, before the ship arrives at the next port. The expected cost calculation of this region is basically the same as region D. By interchanging the first failure time of Engine II, $y$, with $x$ and interchanging the related probabilities in (14), we can calculate the cost incurred in region $\mathrm{E}$.

By using the above equations, we can fully finally calculate the total expected cost from the following integral equation:

$$
\begin{aligned}
V(t)=\mathrm{E}[C(x, y)]= & \int_{Y=t}^{\infty} \int_{X=t}^{\infty} C_{\mathrm{A}} f_{X}(x) f_{Y}(y) \mathrm{d} x \mathrm{~d} y \\
& +\int_{X=t}^{\infty} \int_{Y=0}^{t} C_{\mathrm{B}}(y) f_{X}(x) f_{Y}(y) \mathrm{d} y \mathrm{~d} x \\
& +\int_{Y=t}^{\infty} \int_{X=0}^{t} C_{C}(x) f_{X}(x) f_{Y}(y) \mathrm{d} x \mathrm{~d} y \\
& +\int_{Y=0}^{t} \int_{X=0}^{y} C_{\mathrm{D}}(x) f_{X}(x) f_{Y}(y) \mathrm{d} x \mathrm{~d} y \\
& +\int_{X=0}^{t} \int_{Y=0}^{x} C_{\mathrm{E}}(y) f_{X}(x) f_{Y}(y) \mathrm{d} y \mathrm{~d} x .
\end{aligned}
$$

The above equation can be solved recursively, starting with $t=0$ and increasing $t$ by $\Delta t$, until we reach $t=T_{0}$, the end of operating season. Details of this solution process are given in the next section.

\section{Optimization}

At the end of each operating season, there is a winter layup period, during which some of the engine components may be replaced. The cost of these winter layup actions is denoted as $C_{\mathrm{ws}_{\mathrm{s}}}$ for the one-engine case, and $C_{\mathrm{Wd}}$ for the two-engine case. The winter lay-up cost will be the initial cost of the next operating season. We are looking for the set of winter layup actions which would minimize the expected costs incurred during the next operating season. A combination of the actions taken for each component comprises policy $w$. For $N$ components, there are $2^{N}$ policy alternatives. Each combination of possible winter layup actions taken for system components is defined as a 'set'. We denote each set with superscript $w$.

Equation (3) and (15) can be solved with a recursive procedure. In order to solve these equations recursively, we start with an operating season whose duration, $t$, is zero. We then increase $t$ by $\Delta t$ and solve $V\left(t+\Delta t\right.$ ) from (15) (or $\vartheta_{0}(t)$ from (3) and (4)). $\Delta t$ is an appropriate time step. We keep increasing $t$ and calculating $V(t)$ (or $\left.\vartheta_{0}(t)\right)$ until $t$ reaches $T_{0}$. 
However, only a few integrals of (3) and (15) require recursive iteration for function evaluation. These integrals are given in (16) and (17), for the one- and two-engine cases, respectively.

$$
\begin{aligned}
I_{\mathrm{s}}(t)= & \sum_{j=1}^{N} P_{j} \int_{0}^{t} \mathrm{~d} \xi f_{j}(\xi)\left[\vartheta_{0}\left(t-\xi-l_{j}\right)\right], \\
I_{\mathrm{d}}(t)= & \int_{X=t}^{\infty} \int_{Y=0}^{t} \sum_{j=1}^{N_{I I}} P_{\mathrm{II} j} V\left(\tau_{\mathrm{B}}\right) f_{X}(x) f_{Y}(y) \mathrm{d} y \mathrm{~d} x \\
& +\int_{Y=t}^{\infty} \int_{X=0}^{t} \sum_{i=1}^{N_{\mathrm{I}}} P_{\mathrm{I} i} V\left(\tau_{\mathrm{C}}\right) f_{X}(x) f_{Y}(y) \mathrm{d} x \mathrm{~d} y \\
& +\int_{Y=0}^{t} \int_{X=0}^{y} \sum_{i=1}^{N_{\mathrm{I}}} P_{\mathrm{I} i}\left[\sum_{j=1}^{N_{\mathrm{II}}} P_{\mathrm{II} j} V\left(\tau_{\mathrm{D}}\right)\right] f_{X}(x) f_{Y}(y) \mathrm{d} x \mathrm{~d} y \\
& +\int_{X=0}^{t} \int_{Y=0}^{x} \sum_{j=1}^{N_{\mathrm{II}}} P_{\mathrm{II} j}\left[\sum_{i=1}^{N_{1}} P_{\mathrm{I} i} V\left(\tau_{\mathrm{E}}\right)\right] f_{X}(x) f_{Y}(y) \mathrm{d} y \mathrm{~d} x .
\end{aligned}
$$

We define the partial expected costs, $R_{\mathrm{s}}$ and $R_{\mathrm{d}}$, for the one and two-engine cases respectively, as the following:

$$
\begin{aligned}
& R_{\mathrm{s}}=\vartheta_{0}\left(T_{0}\right)-I_{\mathrm{s}}\left(T_{0}\right), \\
& R_{\mathrm{d}}=V\left(T_{0}\right)-I_{\mathrm{d}}\left(T_{0}\right) .
\end{aligned}
$$

Equations (18) and (19) can be solved by using numerical integration without using recursive iteration.

Our objective is to find the minimum expected cost for the operating season immediately following the winter lay-up, denoted as $C_{t \mathrm{~s}}$ for the one-engine case and as $C_{t \mathrm{~d}}$ for the two-engine case. Our objective functions are

$$
\begin{aligned}
& C_{t \mathrm{~s}}=\min _{w}\left\{C_{\mathrm{Ws}_{\mathrm{s}}}+\vartheta_{0}^{w}\left(T_{0}\right)\right\}, \\
& C_{\mathrm{dd}}=\min _{w}\left\{C_{\mathrm{Wd}}+E^{w}[C(x, y)]\right\},
\end{aligned}
$$

where

$$
\begin{aligned}
\vartheta_{0}^{w}\left(T_{0}\right)= & \sum_{j=1}^{N} P_{j}^{w} R^{w}\left(T_{0}\right)\left[y_{0} T_{0}+\vartheta_{0}^{w}(0)\right] \\
& +\sum_{j=1}^{N} P_{j}^{w} \int_{0}^{t} \mathrm{~d} \xi f_{j}^{w}(\xi)\left[y_{0} \xi+b_{j}+y_{j} l_{j}+\vartheta_{0}^{w}\left(T_{0}-\xi-l_{j}\right)\right]
\end{aligned}
$$

and

$$
E^{w}[C(x, y)]=\int_{0}^{\infty} \int_{0}^{\infty} C^{w}(x, y) f_{X Y}^{w}(x, y) \mathrm{d} x \mathrm{~d} y .
$$

We then calculate the minimum expected cost using the following algorithm:

Step 1. Choose a set of winter actions from all feasible sets and denote it as 'the first set'.

Step 2. Calculate the total expected cost, $C_{t \mathrm{~s}}$ (or $C_{t \mathrm{~d}}$ ), for this selected set using the recursive iteration method explained at the beginning of this section. Denote this cost as the reference total expected cost, $T_{\mathrm{r}}$.

Step 3. Choose another set from available sets. Calculate partial expected cost from equations (18) and (19).

Step 4. Compare partial expected cost with $T_{\mathrm{r}}$. If this partial expected cost is greater than the reference total cost, go to Step 3 and choose another set. Otherwise, go to the next step. 
Step 5. Calculate total expected cost using recursive iteration, then compare this cost with $T_{\mathrm{r}}$. If this cost is less then $T_{\mathrm{r}}$, denote this cost as the new $T_{\mathrm{r}}$ and designate this set as 'the optimal of the evaluated sets'.

Step 6. Continue with Steps 3 through 5, until all feasible sets are exhausted.

The above algorithm has been implemented into computer codes, discussed in the next section. Programs developed using this algorithms provide the decision maker with the necessary tools to assist in maintenance, repair and replacement decisions. Our competing process model enables us to evaluate maintenance requirements for systems composed of a group of components whose failure times are age-dependent.

\section{Implementation and results}

The implementation of the algorithm developed in Section 4 requires solution of (15) for two-engine case. Due to the complexity of the functions in these equations, several integrals are evaluated numerically. We have used the Gauss-Legendre Quadrature method for these numerical integrations.

We have examined several examples using field data. However, we have been unable to gain access to some vital information on actual operating and opportunity costs, treated as proprietary by the company which provided us the failure data. Assumed ranges have been used for these classified costs in our programs. Sensitivity analyses have been performed for several parameters for which we have had insufficient or no information from the industry. We have investigated the influence of parameter variations on our minimum expected cost and the corresponding policies.

We have chosen the Weibull PDF over the exponential PDF for component lifetimes, since it provides a much better fit with our data [7]. For a Weibull PDF with parameters $\alpha$ and $\beta$, the residual reliability becomes

$$
R_{\text {res }}(u)=\exp \left[-\left(\frac{A+u}{\alpha}\right)^{\beta}\right] / \exp \left[-\left(\frac{A}{\alpha}\right)^{\beta}\right]
$$

We developed two computer codes, SEREP (Single Engine Replacement Program) for one-engine systems and DEREP (Double Engine Replacement Program) for two-engine systems. Since the field data that we processed come from three ships equipped with two engines each, we next focus on two-engine systems.

In the following sections, we present the inputs and the results of our program. Different sets of age groups for system components were evaluated in terms of optimal winter layup decisions. Since the field data that we processed come from three ships equipped with two engines each, we only focus on the results of DEREP.

\section{Two-engine case program (DEREP)}

The values of the parameters of (15) are the user inputs required by this program.

We have estimated the Weibull shape and scale parameters for cylinder heads, cylinder jackets, cylinder liners and liner/jacket o-rings, cylinder pistons, fuel cams, turbochargers and connecting bearing rods using actual censored field data. We have also estimated the Weibull shape and scale parameters for the same items. These results are given in Table 1, where $n$ is the number of censored data points observed and $r$ is the number of failures observed [7]. These results are based on data from 1976 to 1988 (included).

We have used the Weibull parameters given in Table 1 on all of our runs. We assumed that all system failures experienced during the operating season cause repairable damages. However, for fixed repair costs, we assumed two different values. Therefore, we introduced two options. Option 1 assumes zero fixed repair costs $\left(b_{j}\right.$ 's) and Option 2 assumes fixed repair costs which are equal to fixed replacement costs. Table 2 shows the values of parameters $l_{j}, W(j), b_{j}$, for our system. 
Table 1

Weibull parameter estimates for selected components

\begin{tabular}{lcrrrr}
\hline Component & $n$ & $r$ & \multicolumn{2}{c}{ Weibull } & \\
\cline { 4 - 5 } & & & $\alpha$ (hrs) & $\beta$ & MTTF (hrs) \\
\hline Connecting bearing rod & 330 & 10 & 31699 & 3.432 & 28494 \\
Cylinder head & 304 & 18 & 69764 & 1.544 & 62766 \\
Cylinder jacket & 290 & 5 & 74802 & 2.195 & 66245 \\
Cylinder liner and o-ring & 321 & 16 & 83769 & 1.424 & 76146 \\
Cylinder piston & 350 & 8 & 211070 & 1.221 & 197688 \\
Fuel cam & 90 & 52 & 60358 & 0.710 & 75396 \\
Turbocharger & 59 & 6 & 31756 & 1.520 & 28625 \\
\hline
\end{tabular}

Port times range between 8 and 16 hours. We have used the lower limit to get a conservative estimate in finding the best replacement policy. We used $50 \mathrm{hrs}$. for voyage times, $T_{l}$ 's. We have examined winter layup choices and associated expected costs for each base by using assumed ranges for operating and opportunity costs.

Only the components which have exactly the same parameters are not treated separately. For example, if piston \#4 and piston \#15 have different ages, they will have to be considered as different components.

As the total number of components considered for replacement increases, the computation time for the minimum expected cost increases exponentially. We have considered seven component types, namely connecting rod bearing, cylinder head, cylinder jacket, cylinder liner and o-ring, cylinder piston, and fuel cam. Each engine has sixteen components for each type we have considered except for turbochargers (two per engine).

Theoretically, we can have as many as sixteen age groups for each of these component types (two for turbochargers). However, for a brand-new ship, we only have one age group, the same for each component type. As the system gets older, the number of age groups generally increases. When extensive replacements are performed in a given year, this number may even decrease in the following year. For a seven or eight year old ship, age groups have been generally observed to be between 1 and 6 per sixteen items in our data.

We have examined replacement policies for a group of 14 components, examining seven components per engine. Although it is not compulsory, we have selected one item from each component type for each engine. (The DEREP program does not require equal number of components from each engine.)

We have chosen two sets of typical age groups for these components taken from 1987 data records. These sets and component identification numbers (ID's) are given in Tables 3 and 4 for ships $A$ and $B$, respectively. If a component type has had more than one age group, we have chosen the oldest age for that component type in these tables. According to the company which provided us the field data, when one engine fails, there is a $36 \%$ decrease in ship speed. Therefore, $\gamma$ is equal to 1.36 for our system. The only change in the operating costs in case of one engine failure is $50 \%$ decrease in fuel costs. Hence, $\eta$ is equal to 0.8 for our system.

Table 2

Data used in examples

\begin{tabular}{lrrr}
\hline Component & $l_{j}$ & $W_{j}$ & $\begin{array}{l}b_{j} \text { for } \\
\text { replacement }\end{array}$ \\
\hline Connecting bearing rod & 16 & 400 & 400 \\
Cylinder head & 8 & 20000 & 20000 \\
Cylinder jacket & 16 & 10000 & 10000 \\
Cylinder liner and o-ring & 16 & 10300 & 10300 \\
Cylinder piston & 16 & 3600 & 3600 \\
Fuel cam & 16 & 3000 & 3000 \\
Turbocharger & 30 & 29700 & 27000 \\
\hline
\end{tabular}


Table 3

Component ages for ship $A$

\begin{tabular}{lllcc}
\hline Component & ID & Eng. I- $A(j)$ & ID & Eng. II- $A(j)$ \\
\hline Connecting bearing rod & 1 & 14534 & 8 & 19445 \\
Cylinder head & 2 & 27603 & 9 & 27573 \\
Cylinder jacket & 3 & 14534 & 10 & 14527 \\
Cylinder liner and o-ring & 4 & 14534 & 11 & 14527 \\
Cylinder piston & 5 & 14534 & 12 & 14527 \\
Fuel cam & 6 & 27603 & 13 & 27573 \\
Turbocharger & 7 & 5050 & 14 & 5030 \\
\hline
\end{tabular}

Table 4

Component ages for ship $B$

\begin{tabular}{lllcc}
\hline Component & ID & Eng. I- $A(j)$ & ID & Eng. II- $A(j)$ \\
\hline Connecting bearing rod & 1 & 15656 & 8 & 10136 \\
Cylinder head & 2 & 11218 & 9 & 29034 \\
Cylinder jacket & 3 & 11218 & 10 & 36413 \\
Cylinder liner and o-ring & 4 & 10121 & 11 & 36413 \\
Cylinder piston & 5 & 8746 & 12 & 10136 \\
Fuel cam & 6 & 34422 & 13 & 5157 \\
Turbocharger & 7 & 5149 & 14 & 7194 \\
\hline
\end{tabular}

\subsection{Contributions of different regions to the total expected cost}

The portions of the expected cost incurred from regions A, B, C, D, E, shown in Figure 1 and are denoted with $A_{\mathrm{c}}, B_{\mathrm{c}}, C_{\mathrm{c}}, D_{\mathrm{c}}, E_{\mathrm{c}}$, respectively. These costs are calculated from equations (24)-(28), respectively.

$$
\begin{aligned}
& A_{\mathrm{c}}=\int_{Y=t}^{\infty} \int_{X=t}^{\infty} C_{\mathrm{A}} f_{X}(x) f_{Y}(y) \mathrm{d} x \mathrm{~d} y ; \\
& B_{\mathrm{c}}=\int_{X=t}^{\infty} \int_{Y=0}^{t} C_{\mathrm{B}}(y) f_{X}(x) f_{Y}(y) \mathrm{d} y \mathrm{~d} x ; \\
& C_{\mathrm{c}}=\int_{Y=t}^{\infty} \int_{X=0}^{t} C_{\mathrm{C}}(x) f_{X}(x) f_{Y}(y) \mathrm{d} x \mathrm{~d} y ; \\
& D_{\mathrm{c}}=\int_{Y=0}^{t} \int_{X=0}^{y} C_{\mathrm{D}}(x) f_{X}(x) f_{Y}(y) \mathrm{d} x \mathrm{~d} y ; \\
& E_{\mathrm{c}}=\int_{X=0}^{t} \int_{Y=0}^{x} C_{\mathrm{E}}(y) f_{X}(x) f_{Y}(y) \mathrm{d} y \mathrm{~d} x .
\end{aligned}
$$

We have investigated the magnitude of the costs associated with regions $\mathrm{A}, \mathrm{B}, \mathrm{C}, \mathrm{D}$ and $\mathrm{E}$. We have expected that costs incurred from regions $\mathrm{D}$ and $\mathrm{E}$ to be significantly smaller than those of other regions. For our parameters, $A_{\mathrm{c}}$ comprises more than $80 \%$ of the total costs whereas the expected costs of regions $\mathrm{D}$ and $\mathrm{E}$ constitute less than $1 \%$ of the total expected costs. On the other hand, about $6-10 \%$ of the total cost comes from regions $\mathrm{B}$ and $\mathrm{C}$. The cost calculation of regions $\mathrm{D}$ and $\mathrm{E}$ has proved to be much more time consuming than those of other regions. By considering both the small magnitude of region $\mathrm{D}$ and $\mathrm{E}$ expected costs and very large running time required to calculate these costs, we have decided to neglect regions $\mathrm{D}$ and $\mathrm{E}$ in our later runs. However, we have seen that the magnitude of region $\mathrm{D}$ and $\mathrm{E}$ costs could become significant as the ages of system components and the length of operating season substantially increase. 
Table 5

Sensitivity analysis results

\begin{tabular}{lrrllll}
\hline Run no. & $Y_{0}$ & $Y_{\mathrm{oc}}$ & Option & Age set & $C_{\mathrm{td}}$ & Corresponding policy \\
\hline 1 & 375 & 950 & 1 & B & 1495936 & Keep all components \\
2 & 750 & 1900 & 1 & B & 2990259 & Keep all components \\
3 & 1500 & 3800 & 1 & B & 5974659 & Replace 6, 8, 13, keep others \\
4 & 375 & 3800 & 1 & B & 1503586 & Keep all components \\
5 & 375 & 950 & 2 & A & 1490889 & Keep all components \\
6 & 750 & 1900 & 2 & A & 2980957 & Replace 6, keep others \\
7 & 1500 & 3800 & 2 & A & 5958181 & Replace 6, 13, keep others \\
8 & 750 & 1900 & 2 & B & 2988737 & Keep all components \\
9 & 375 & 950 & 2 & B & 1494414 & Keep all components \\
10 & 375 & 3800 & 2 & B & 1502065 & Keep all components \\
11 & 750 & 1900 & 1 & A & 2982735 & Replace 6, keep others \\
12 & 1500 & 3800 & 1 & A & 5960002 & Replace 6, 13, keep others \\
13 & 1500 & 3800 & 2 & B & 5972772 & Replace 6, 8, 13, keep others \\
\hline
\end{tabular}

\subsection{Best replacement policies for two-engine systems}

Next, we have examined the minimum expected cost and the corresponding policies, for two ships with two fixed repair cost possibilities denoted as options, and three different opportunity and operating cost sets. One input set represents the replacement policies for the average values of these costs. Two other input sets represent the best replacement policies for the assumed extreme values of these costs. Our results for these selected sets are given in Tables 5 and 6.

When we double and quadruple both the opportunity and operating costs as in samples 1-3 and 6-9 of Table 5, the total expected cost almost doubles and quadruples, respectively. Therefore, the expected cost is again very sensitive to operating costs. These results have also showed that even for the extreme values of operating costs the minimum expected cost is obtained by keeping (not replacing) almost all items considered for winter replacement.

This expected result is a consequence of the current conservative replacement practices. We observed that the difference in the expected cost for the best and the worst replacement policies could be as high as $\$ 150000$, per ship, per operating season. We examined the best replacement policy and the ones immediately following the best policy in terms of expected costs. Table 7 shows the total expected costs for top ten policies and the worst policy for different parameters. In this table, runs 1, 2, 3, 4 refer to runs 10 , 11,12 and 2 of Table 5 .

Table 6

Distribution of partial expected costs

\begin{tabular}{rlrrrr}
\hline Run No. & $A_{\mathrm{c}}$ & $B_{\mathrm{c}}$ & $C_{\mathrm{c}}$ & $C_{\mathrm{Wd}}$ & $C_{\mathrm{d}}$ \\
\hline 1 & 1229556 & 89922 & 106456 & 0 & 1495936 \\
2 & 2599113 & 179029 & 212116 & 0 & 2990259 \\
3 & 5082520 & 410106 & 475632 & 6400 & 5974659 \\
4 & 1299556 & 93417 & 110612 & 0 & 1503586 \\
5 & 1249547 & 148554 & 92787 & 0 & 1490889 \\
6 & 2471383 & 293672 & 212900 & 3000 & 2980957 \\
7 & 4908563 & 620880 & 422738 & 6000 & 5958181 \\
8 & 2599112 & 178256 & 211368 & 0 & 2988737 \\
9 & 1299556 & 89150 & 105708 & 0 & 1494414 \\
10 & 1299556 & 92644 & 109863 & 0 & 1502065 \\
11 & 2471384 & 294646 & 213704 & 3000 & 2982735 \\
12 & 4908563 & 621899 & 423539 & 6000 & 5960002 \\
13 & 5082520 & 409062 & 474790 & 6400 & 5972772 \\
\hline
\end{tabular}


Table 7

Ranking of total expected costs

\begin{tabular}{lllll}
\hline Rank & Run 1 & Run 2 & Run 3 & Run 4 \\
\hline 1 & 1502065 & 2982735 & 5960002 & 2990259 \\
2 & 1502536 & 2983368 & 5960589 & 2990644 \\
3 & 1503556 & 2983964 & 5963483 & 2990996 \\
4 & 1504075 & 2984813 & 5963496 & 2991154 \\
5 & 1504449 & 2986281 & 5964077 & 2991240 \\
6 & 1504479 & 2986292 & 5964099 & 2991344 \\
7 & 1504786 & 2986434 & 5964795 & 2991528 \\
8 & 1504932 & 2986932 & 5964950 & 2991714 \\
9 & 1505623 & 2986935 & 5966977 & 2993846 \\
10 & 1505663 & 2987506 & 5967273 & 2993850 \\
Worst & 1653688 & 3148579 & 6146768 & 3148700 \\
\hline
\end{tabular}

Table 8

Extreme case results

\begin{tabular}{rllll}
\hline$Y_{0}$ & $Y_{\mathrm{oc}}$ & Case & $C_{\mathrm{d}}$ & Best set \\
\hline 750 & 1900 & 2 & 2476502 & Replace only 2, 3, 4, 7, 9, 10, 11, 14 \\
1500 & 3800 & 2 & 4812881 & Replace only 2, 3, 4, 7, 9, 10, 11, 14 \\
\hline
\end{tabular}

Differences among these expected costs are less than $\$ 500$ for some cases within our parameter range. This amount constitutes less than $0.01 \%$ of the total expected cost. Considering the approximations used, regions ignored and the censored nature of the data we have processed, these differences are not significant.

It is interesting to investigate what would be the best replacement policy if none of the items has been replaced until the current replacement period; e.g., the ages of system components equal to $36413 \mathrm{hrs}$. (assuming nothing failed until the current winter layup). Results for such a set, shown in Table 8, indicate that more than half of system components should be replaced in order to minimize expected costs. Hence, the best replacement policy is also rather sensitive to component ages.

\section{Concluding remarks}

We have developed reliability-based replacement models to enhance current winter layup practices of Great Lakes marine diesels. We implemented and extended Howard's one-set competing process model, which is applicable to ships with one engine on board, to a two-set competing process model suitable for two-engine ships. Unlike many existing models, our models incorporate the age-dependent nature of component failure behavior.

Using the above models, we have developed computer codes producing rational winter layup replacement policies. Programs SEREP and DEREP produce the best replacement policies for a group of selected system components for one- and two-engine systems. We have examined several examples using field data. We observed that there is a group of 'good' replacement policies immediately following the best replacement policy; differences between the total expected costs resulting from these policies are not very significant for some cases within our parameter range.

It is our belief that our codes would be helpful to all shipping companies with diesel-powered ships, and primarily most Great Lakes fleets. Our results could also be of interest to various ship design firms, as well as classification societies (ship registers), aiding them in deciding on the power plant configuration and the optimal number of stand-by and redundant components. Clearly, our findings do not merely apply to 
ships; diesel engines aboard any other systems can be analyzed similarly and optimal maintenance, repair and replacement policies could be obtained with some modifications.

The benefits would start with more reliable average failure and replacement interval estimates, which would translate to more realistic replacement policies that will not jeopardize the objective of no failures during the operating season. The trial of the new replacement policies will create operational experience which could allow feedback to update the engine manufacturer's typically quite conservative replacement recommendations. Regulatory agencies may later also consider changing some of their recommendations regarding the determination of periodic survey intervals and the like.

\section{Appendix A. List of symbols}

$b_{j}=$ Fixed cost incurred immediately after component $j$ fails $(\$)$,

$E(\cdot)=$ Expected value;

$C(x, y)=$ Cost incurred during an operating season by a two-engine system (\$);

$f_{j}(\cdot)=$ Holding time PDF of a one-engine system making a transition to down state $j$, after a competition among $N$ down states, in accordance with the competing process model;

$F(\cdot)=$ Cumulative distribution function (CDF);

$g_{j}(\cdot)=$ Residual lifetime PDF of a component whose failure will bring an engine to down state $j$;

$l_{j}=$ TTR (Time to repair or replacement) of failed engine component: time required to bring back the failed engine to its operating condition, starting at its failure, by means of a repair (or replacement) process (hrs);

$l_{j}^{\prime}=$ Time required to complete repairs of component $j$, in addition to scheduled port stay (hrs);

$L_{\text {op }}=$ Fixed travel time between two consecutive ports (hrs);

$N_{\mathrm{I}}=$ Number of down states of Engine I;

$N_{\text {II }}=$ Number of down states of Engine II;

$P_{j}=$ Transition probability of an operating engine for going to down state $j$;

$\mathrm{PDF}=$ Probability density function;

$\mathrm{R}(\cdot)=$ Reliability function;

$\mathrm{rpm}=$ Revolutions per minute;

$t_{p}=$ Departure time from the previous port (hrs);

$t_{p 0}=$ Scheduled arrival time at the next port given failure-free operation (hrs);

$t_{p 1}=$ Delayed arrival time at the next port given that one of the engines of a two-engine system fails during the voyage (hrs);

$t_{p 2}=$ Delayed arrival time at the next port given that both Engine I and Engine II fail during the voyage (hrs);

$t_{\mathrm{r}}=$ Completion time for repair at sea (hrs);

$T_{0}=$ Total system operating time during the season under consideration (hrs);

$T_{i}=$ Length of fixed travel time between two consecutive ports (hrs);

$V(t)=$ Expected total cost that a two-engine system will incur for an operating season of length $t$ after it starts operating at time zero $(\$)$;

$V(0)=$ Terminal costs that a two-engine system will incur if it is operational at the end of an operating season (\$);

$X=$ First failure time of Engine $\mathrm{I}(\mathrm{hrs})$;

$Y=$ First failure time of Engine II (hrs);

$y_{j}=$ Occupancy cost per unit residence time incurred during an engine's stay at failure state $j(\$ / \mathrm{hrs})$;

$y_{j}^{*}=$ Repair cost per unit residence time incurred during an engine's stay at failure state $j(\$ / \mathrm{hrs})$;

$y_{0}=$ Constant operating cost for one engine $(\$ / \mathrm{hrs})$;

$Y_{j}=$ Variable repair cost per unit residence time incurred during the system's stay at failure state $j$ in two engine case $(\$ / \mathrm{hrs})$;

$Y_{\mathrm{c}}=$ Opportunity cost per unit residence time $(\$ / \mathrm{hrs})$;

$Y_{0}=$ Constant operating cost of a system per unit residence time $(\$ / \mathrm{hrs})$; 
$\alpha=$ Scale parameter of a Weibull PDF;

$\beta=$ Shape parameter of a Weibull PDF;

$\gamma=$ Ratio of the ship speed when both engines are operating over the ship speed when one engine is operating $(\gamma>1)$;

$\eta=$ Ratio of operating costs for one-engine operation over those for two-engine operation for a two-engine system $(\eta<1)$;

$\vartheta_{0}(t) \equiv$ Expected total cost that a one-engine system will incur by time $t$ (given it starts operating at time zero) (\$);

$\vartheta_{0}(0) \equiv$ Terminal costs that a one-engine system will incur if it occupies state 0 at time $T_{0}(\$)$.

\section{Acknowledgement}

We would like to thank the Michigan and National Sea Grant College Programs for the funding of a three-year research project directed by the first author. We would also like to thank the management and the technical staff of Interlake Steamship, especially fleet engineer Mr. Richard Harkins, for their valuable inputs and for allowing us to use their (annually updated) reliability/replacement database. Professor M.G. Parsons' and S.M. Pollock's inputs to this project are also appreciated.

\section{References}

[1] Howard, R., Dynamic Probabilistic Systems, Volumes I and II, Wiley, New York, 1971.

[2] İnözü, B., and Perakis, A.N., "Reliability-based replacement algorithms and applications to marine diesels', presented at the Operations Research 1990 International Conference, Vienna, Austria, August 28-31, 1990, and accepted for inclusion in the Proceedings of the German Mathematical Operations Research Societies (GMÖOR) Springer-Verlag, July 1990.

[3] Nikolaidis, E., and Parsons, M.G., Marine diesel maintenance management system for the Interlake Steamship Company, Unpublished Report, 1984.

[4] Perakis, A.N., and İnözü, B., "Reliability analysis of marine diesels: State-of-the-Art and current modeling", Marine Technology 27/4, 237-249 (1990).

[5] Perakis, A.N., and İözü, B., "Reliability analysis of Great Lakes marine diesels: Toward rational maintenance, repair and replacement decisions", Interim Report, Michigan Sea Grant Program, The University of Michigan, October 1988.

[6] Ross, S.H., Stochastic Processes, Wiley, New York, 1983.

[7] İnözü, B., and Perakis, A.N., "Statistical analysis of failure time distributions for Great Lakes marine diesels using censored data," Journal of Ship Research 35/1, 73-82 (1991). 